\title{
Hybrid FPGA/ARM Co-design for Near Real Time of Remote Sensing Imagery
}

\author{
C. Góngora-Martín ${ }^{1, \star}$, A. Castillo-Atoche ${ }^{1}$, \\ J. Estrada-López ${ }^{1}$, J. Vázquez-Castillo ${ }^{2}$, \\ J. Ortegón-Aguilar ${ }^{2}$, and R. Carrasco-Álvarez ${ }^{3}$ \\ 1 Universidad Autónoma de Yucatán, Mérida, México \\ \{acastillo, johan.estrada\}@uady.mx \\ 2 Universidad de Quintana Roo, Chetumal, México \\ \{jvazquez, jortegon\}@uqroo.mx \\ 3 Universidad de Guadalajara, Guadalajara, México \\ roberto.carrasco@red.cucei.udg.mx
}

\begin{abstract}
A novel hybrid co-design for implementing high-resolution reconstruction algorithms, for near real time implementation of remote sensing (RS) imagery, is addressed in this paper. In the proposed codesign scheme, the inverse square root and the matrix operations of the robust adaptive space filter algorithm are implemented as accelerators units in a Field Programmable Gate Array (FPGA) using piecewise polynomial approximations and systolic array (SA) techniques. Then, the FPGA based accelerator is integrated with an ARM processor in a $\mathrm{HW} / \mathrm{SW}$ co-design paradigm that meets the (near) real time imaging systems requirements in spite of conventional computations. Finally, we report and discuss the results of the hybrid FPGA/ARM co-design implementation in a Xilinx Virtex-5 XC5VFX70TFFG1136 for reconstruction of real world RS images.
\end{abstract}

Keywords: Remote sensing, systolic arrays, HW/SW co-design.

\section{Introduction}

Developing computationally efficient processing techniques for massive volumes of remote sensing data is critical for space-based Earth science and planetary exploration (see for example, 1], 2] and the references therein). In particular, with the advent of new remote sensing missions and their increased temporal resolutions, the availability and dimensionality of data is continuously increasing. This demands fast processing solutions that can be used to compress and/or interpret remote sensing data onboard spacecraft imaging platforms. In adition, a response in near real time is required in areas such as environmental modeling and assessment, target detection for military and homeland defense/security purposes, and risk prevention and response.

\footnotetext{
* Corresponding author.
} 
To address the computational requirements introduced by many time-critical applications, several research efforts have been recently directed towards the incorporation of parallel computing techniques and the design of specialized hardware accelerators architectures. In this regards, reconfigurable hardware solutions such as field-programmable gate arrays (FPGAs) have been consolidated as the standard choice for onboard remote sensing processing due to their smaller size, weight, and power consumption when compared with other high-performance computing systems, such as Graphic Processor Units (GPUs), digital signal processors (DSPs) or inclusive clústers of personal computers. Although there have been many literature sources on the use of FPGAs in remote sensing, in general, there still a challenge to design specialized hardware accelerators units in a HW/SW co-design scheme for being implemented to such missions. The principal innovative proposition that distinguishes our approach from the previous studies [3], 4] consists of a hybrid FPGA/ARM co-design aimed at the digital implementation of the Robust Adaptive Space Filter (RASF) regularization technique in a computationally efficient fashion that meets the near real time imaging system requirements. Our strategy for implementing the reconstructive signal processing architectures in a FPGA/ARM platform is aimed at enhancing the locality through the utilization of HPEC techniques to precisely represent loop programs and to compute complex sequences of loop transformations (interchange, fusion, fission, skewing, tiling, etc.) while preserving the original program semantics and also, by mapping the transformed algorithmic representation in systolic arrays (SAs). The inverse of a $n \times n$ matrix is based on the QR decomposition via the Givens rotation method. Then, the inverse square root arithmetic function $1 / \sqrt{x}$ is implemented in HW using systolic arrays and piecewise polynomial approximation techniques, which allows to achieve a maximum level of parallelism.

\section{Summary of the RASF Regularization Algorithm}

The general mathematical formalism of the RS problem and the DEDR-RASF regularization algorithm is presented in this section. Some crucial elements that we employ in this paper are similar in notation and structure to that described in [1], 3], however, this is necessary for the understanding of the readers.

Let us consider the estimation of the scene pixel-frame image $\hat{\mathbf{B}}$ via lexicographical reordering $\hat{\mathbf{B}}=L\{\hat{\mathbf{b}}\}$ of the spatial spectrum pattern (SSP) vector estimate $\hat{\mathbf{b}}$ reconstructed from whatever available measurements of independent realizations $\{u(j) ; j=1, \ldots, J\}$ of the recorded RS data field. The measurement data wavefield $u(\mathbf{y})=s(\mathbf{y})+n(\mathbf{y})$ is modelled as a superposition of the echo signals $s$ and additive noise $n$ that assumed to be available for observations and recordings within the prescribed time-space observation domain $\mathrm{Y} \ni \mathbf{y}$, where $\mathbf{y}$ $=(\mathbf{t}, \mathbf{p})^{T}$ defines the time-space points in the observation domain $Y=T \times P$, with $T$ being the time and $P$ being the sensor position. The conventional finitedimensional vector-form approximation form of the RS data observation model is given by [1]. 


$$
\mathbf{u}=\mathbf{S e}+\mathbf{n}
$$

where $\mathbf{u}, \mathbf{n}$ and $\mathbf{e}$ define the vectors composed of the coefficients of the finite dimensional approximations of the measurement field $u$, the observation noise $n$ and the scene scattered field $e$, respectively, and $\mathbf{S}$ is the matrix-form approximation of the signal formation operator ( $\mathrm{SFO}$ ) $\mathbf{S}$ specified by the particular modulation format employed in the RS system in [1, 3. The second order statistics $\mathbf{b}=\operatorname{vect}\left\{<e_{k}, e_{k^{*}}>; k=1, \ldots, K\right\}$ of the random scene scattering vector $\mathbf{e}$ has a statistical meaning of the average power scattering function traditionally referred to as the spatial spectrum pattern (SSP) of the RS scene [2]. The SSP represents the brightness reflectivity of the scene image $\mathbf{B}=L\{\mathbf{b}\}$, over the rectangular scene frame in a conventional pixel format.

The RS imaging problem is stated as follows: to find an estimate of the scene pixel-frame image $\hat{\mathbf{B}}$ via lexicographical reordering $\hat{\mathbf{B}}=L\{\hat{\mathbf{b}}\}$ of the spatial spectrum pattern (SSP) vector estimate $\hat{\mathbf{b}}$ reconstructed from whatever available measurements of independent realizations $\{u(j) ; j=1, \ldots, J\}$ of the recorded data vector.Thus, one can seek to estimate, $\hat{\mathbf{b}}$, as a discrete-form representation of the desired SSP, given the data correlation matrix $\mathbf{R}_{\mathbf{u}}=\mathbf{Y}$ pre-estimated empirically via averaging $J \geq 1$ recorded data vector snapshots $\{u(j)\}$ [1]; and by determining the solution operator that we also refer to as the signal formation operator (SFO) $\mathbf{F}$ such that

$$
\hat{\mathbf{b}}=\left\{\hat{\mathbf{R}}_{e}\right\}_{\text {diag }}=\left\{\mathbf{F Y} \mathbf{F}^{+}\right\}_{\text {diag }}=\left\{\mathbf{F u u}^{+} \mathbf{F}^{+}\right\}_{\text {diag }}
$$

where $\{\cdot\}$ diag defines the vector composed of the principal diagonal of the embraced matrix. To optimize the search of such SFO F, we formulate here the following regularization strategy [1]

$$
\mathbf{F} \rightarrow \min _{F}\{\mathfrak{R}(\mathbf{F})\}
$$

where

$$
\mathfrak{R}(\mathbf{F})=\left\{\operatorname{trace}(\mathbf{F S}-\mathbf{I}) \mathbf{A}(\mathbf{F S}-\mathbf{I})^{+}\right\}+\alpha \operatorname{trace}\left\{\mathbf{F R}_{n} \mathbf{F}^{+}\right\}
$$

implies the minimization of the weighted sum of the systematic and fluctuation errors in the desired estimate $\hat{\mathbf{b}}$ where the selection (adjustment) of the regularization parameter $\alpha$ and the weight matrix $\mathbf{A}$ provide the additional experiment design degrees of freedom incorporating any descriptive properties of a solution if those are known a priori [1, 3.

Solving the minimization problem of (3), we obtain the desired DEDR-optimized SFO as follows

$$
\mathbf{F}_{\mathrm{DEDR}}=\mathbf{K}_{\mathbf{A}, \alpha} \mathbf{S}^{+} \mathbf{R}_{n}{ }^{-1}
$$

where

$$
\mathbf{K}_{\mathbf{A}, \alpha}=\left(\mathbf{S}^{+} \mathbf{R}_{n}{ }^{-1} \mathbf{S}+\alpha \mathbf{A}^{-1}\right)^{-1}
$$

defines de so-called reconstruction operator. Note that in practical RS scenarios, the additive observation noise power is adopted for the noise correlation matrix 
$\mathbf{R}_{n}=N_{0} \mathbf{I}[2]$. Considering these assumptions, the robust regularization-related SFO becomes

$$
\mathbf{F}=\left(\mathbf{\Psi}+\alpha N_{0} \mathbf{A}^{-1}\right)^{-1} \mathbf{S}^{+}=\mathbf{K} \mathbf{S}^{+}
$$

with the discrete-form ambiguity function matrix operator $\boldsymbol{\Psi}=\mathbf{S}^{+} \mathbf{S}$. Furthermore, we can adjust the processing level degrees of freedom $\left\{\alpha, N_{0}, \mathbf{A}\right\}$ which specify a family of relevant regularization-related techniques as follows

$$
\hat{\mathbf{b}}=\left(\mathbf{F u u} \mathbf{F}^{+}\right)_{\operatorname{diag}}=(\mathbf{F u}) \circ(\mathbf{F u})^{+}
$$

where o defines the Shur-Hadamar (element by element) vector-matrix product and $\mathbf{F}$ represents represents the conventional reconstructive operator. In this study, the parameters $\alpha$ and $\mathbf{A}$ are adjusted in an adaptive fashion following the Bayesian minimum risk strategy [1]; i.e., $\alpha \mathbf{A}^{-1}=\hat{\mathbf{D}}^{-1}=\operatorname{diag}^{-1}(\hat{\mathbf{b}})$, in which case the SFO (7) becomes a solution-dependent adaptive operator which corresponds to the Robust Adaptive Space Filter (RASF) algorithm.

$$
\mathbf{F}_{\mathrm{RASF}}=\left(\mathbf{\Psi}+N_{0} \hat{\mathbf{D}}^{-1}\right)^{-1} \mathbf{S}^{+}
$$

\section{Efficient FPGA/ARM Co-design of the RASF Algorithm}

In this section, the specific hybrid FPGA/ARM co-design for implementing the RASF algorithm is presented. The co-design methodology is the following. First, the RASF algorithm is adapted in a co-design scheme applying HPEC techniques, and then, the selected computationally complex reconstructive operations are efficiently implemented in hardware accelerator architecture. In the design, we propose to use the FPGA as a high-speed reconfigurable platform and the Beagle Bone Black platform, which is an embedded Linux development board with a Texas Instrument's Sitara AM3359 Cortex-A8 processor is selected for the SW operations. The main parameters to consider in the partitioning stage are the task execution speed and the area required by its HW-level implementation. Based on those parameter considerations, the hybrid co-design is carried out, which consists in deciding which tasks should be executed in SW and which one should be implemented by HW. Another challenging task of the co-design is to manage the large block of data avoiding unnecessary data transfer from/to the ARM processor to/from the proposed systolic HW accelerator. To implement the fixed-point RASF algorithm, we first specify the corresponding computational procedures in the rectangular scene frame $r=(x, y) \in \mathbf{R}$ Such multi-stage procedures are formalized in the following algorithmic scheme as follows 


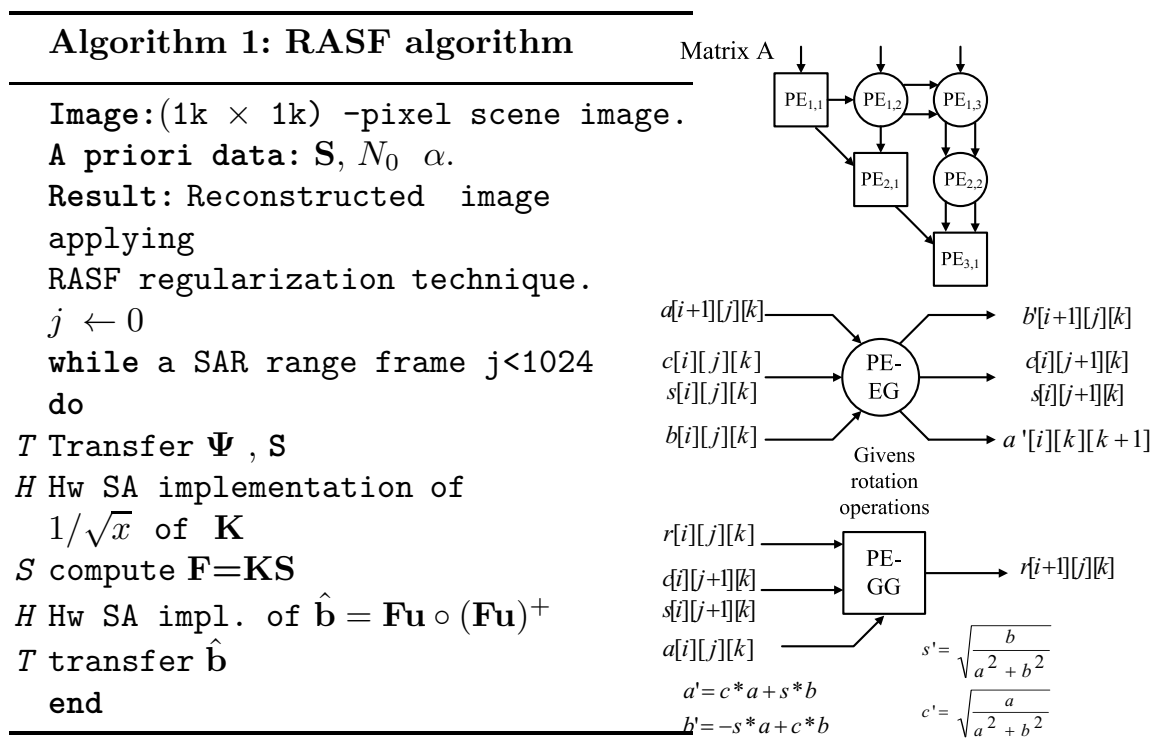

Fig. 1. Hybrid design of the matrix inverse algorithm

The algorithm above shows an outline of the proposed method. A label indicating the $\mathrm{SW} / \mathrm{HW}$ operation precedes each computation operation. The label $\mathrm{T}$ indicates the transfer to/from the embedded processor from/to the SA architecture. The label $\mathrm{H}$ represents the operations implemented by the SAs and label $\mathrm{S}$ the corresponding operations executed by the embedded processor.

\subsection{Embedded ARM Processor}

In this subsection, we describe the implementation of the SW-operations of the RASF algorithm with an embedded processor. According to algorithm 1, the more computationally complex operation is the inverse matrix. For the inverse matrix operation, let us consider the QR transformation of an $m \times n$ matrix $\mathbf{A}$ of full rank $n$ into a triangular matrix $\mathbf{R}$ and an orthogonal matrix $\mathbf{Q}$. Then, finding the pseudo-inverse of matrix $\mathbf{A}$, it is equal to.

$$
\mathbf{A}^{-1}=\left(\mathbf{A}^{T} \mathbf{A}\right)^{-1} \mathbf{A}^{T}=\left(\mathbf{R}^{T} \mathbf{R}\right)^{-1} \mathbf{R}^{T} \mathbf{Q}^{T}=\mathbf{R}^{-1} \mathbf{Q}^{T}
$$

where $(\cdot)^{T}$ is the transpose operation of a matrix.

The Givens Rotation technique consists in solving QR by applying a series of rotations to the rows of the original matrix. That is, this technique determine the upper triangular matrix $\mathbf{R}$, with the proper calculation of parameters $c$ (i.e., cosine), $s$ (i.e., sine) and $r$, As can be seen from figure 1, two different processing elements, which corresponds to Givens Generation and Rotation operations are defined. Likewise, it is pointed out that inverse square root operation is the most computationally complex operation included in the inverse matrix algorithm. Therefore, in this study we address the design of the matrix inverse 
module following the hybrid FPGA/ARM architecture based on the HW/SW co-design paradigm as follows: a HW-core, for concurrent computing of the fixedpoint inverse square root, and a SW-core, for computing sequentially the rest of the QR decomposition operations. Now, we proceed to transform the QR decomposition source program into a parallel algorithm representation. Here, the following parallel computing techniques were used: code sinking (transformation of non-perfectly nested loops), normalization (positive index increment), index matching, single assignment and broadcast removal. Analyzing the dependence graph shown in figure 1, we can notice that the operations in the Generation of Givens (GG) Rotation section, involve the inverse square root, which is computationally complex to be implemented sequentially in SW. Thus, we design in this study an efficient hardware co-processor using piecewise polynomial approximation and systolic arrays techniques in order to accelerate the time processing of the algorithm.

\subsection{FPGA-Based Hardware Co-processors}

In this subsection, we propose the SA-based HW architectures for the fixedpoint RASF algorithm. Typical hardware arithmetic implementations are based on LUTs. In this study, the inverse square root co-processors shown in figure 2(a) and the RASF estimator shown in figure 2(b) are designed. The first one is implemented with the aggregation of piecewise polynomial approximation (typically known as splines) and the second one via systolic array techniques.

The design specification of the inverse square root co-processor is the following: the end user is able to select the number of $m$ segments and the degree $\mathrm{d}$ of
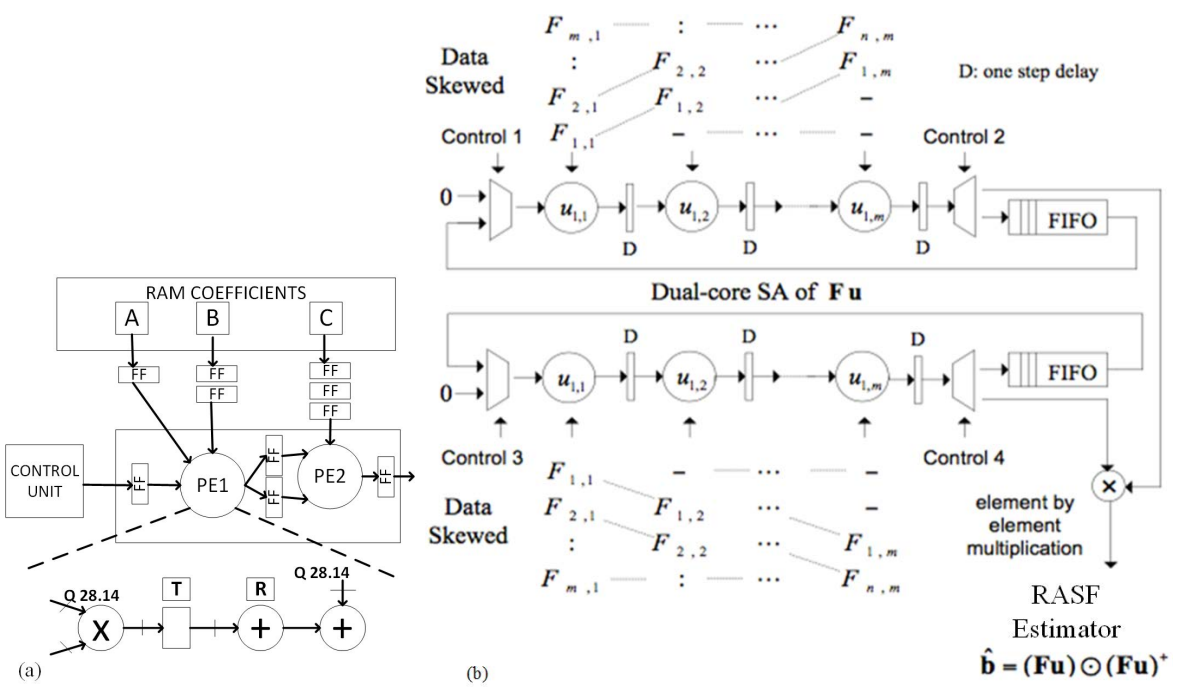

Fig. 2. Hardware coprocessors: (a) Inverse square root design, and (b) Dual-core RASF estimator 
the polynomials related to each segment (spline). A non-uniform segmentation scheme is considered in order to reduce the area of the design having the same accuracy as we consider uniform segmentation. The hardware blocks of the inverse square root architecture can be seen in figure 2(a). The dual-core SA-based RASF estimator is implemented concurrently as follows: the $\mathbf{F u}$ matrix-vector operations are computed for each row of the degraded RS image. Next, both results are multiplied element by element in a parallel architecture as shown in figure 2(b). The resulting SA architecture performs the discrete-form representation of the desired SSP in a high-performance structure. Once the SAs of the RASF algorithm have been defined, we are ready to integrate the design with the hybrid FPGA/ARM approach.

\section{Results and Performance Analysis}

In the RASF algorithmic implementation via the hybrid FPGA/ARM co-design scheme using SAs accelerators, we report the enhancement of the real-world RS images acquired with different fractional SAR systems characterized by the PSF of a Gaussian "bell" shape in both directions of the 2-D scene (in particular, of 16 pixel width at 0.5 from its maximum for the $1 K$-by- $1 K$ BMP pixel-formatted scene). In analogy to the image reconstruction, we employed the quality metric defined as an improvement in the output signal-to-noise ratio (IOSNR) [2]. The initial test scene is displayed in figure 3(a). figure 3(b) presents the same original image but degraded with the MSF method. The qualitative HW results for the RSF and RASF enhancement/reconstruction procedures are shown in figures [3](c) and 3](d) with the corresponding IOSNR quantitative performance enhancement metrics reported in the figure captions (in the $[\mathrm{dB}]$ scale).

The synthesis metrics related to the implementation of the $\mathrm{HW} / \mathrm{SW}$ co-design RASF algorithm is summarized in Table 1 . These metrics specify the area and time behaviors of the corresponding hardware, i.e. the RASF estimator and the inverse square root coprocessors. Last, it is compared the achieved processing

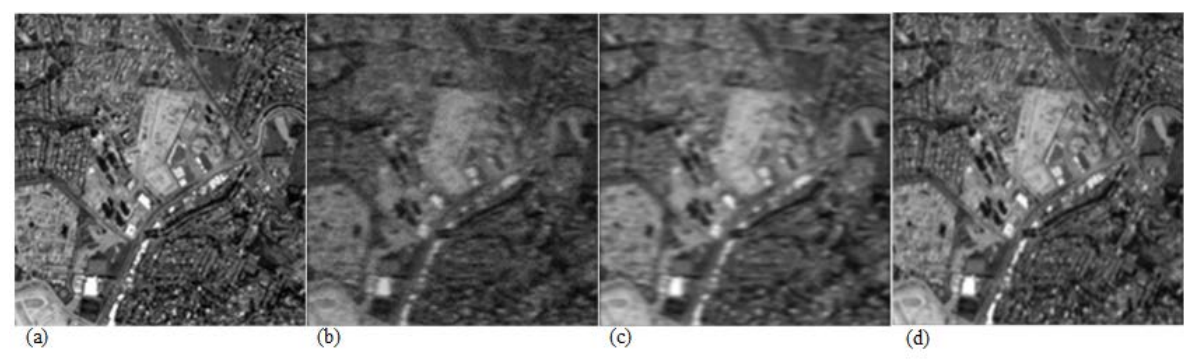

Fig. 3. Implementation results: (SNR $u=15 \mathrm{~dB}$ ): (a) original test scene; (b) degraded scene image formed applying the MSF method; (c) image reconstructed applying the RSF algorithm (IOSNR $=7.49 \mathrm{~dB})$; $(\mathrm{d})$ image reconstructed applying the RASF algorithm (IOSNR $=9.14 \mathrm{~dB})$. 
time of the RASF algorithm using the HW/SW co-design paradigm as reported in Table 2. In the reported analysis of Table 2, we consider to use a personal computer (PC) running at $3 \mathrm{GHz}$ with AMD Athlon (tm) 64 dual-core processor and $2 \mathrm{~GB}$ of RAM memory for the conventional MATLAB and $\mathrm{C}++$ reference implementations.

Table 1. Synthesis Metrics

Table 2. Timeprocessing Comparative

\begin{tabular}{|c|c|c|c|c|}
\hline $\begin{array}{l}\text { SA accelerator } \\
\text { Accelerator }\end{array}$ & $\begin{array}{l}\text { RASF } \\
\text { Estimator }\end{array}$ & $\begin{array}{c}\text { Inverse } \\
\text { Square Root }\end{array}$ & Method $\rightarrow$ & $\begin{array}{c}\text { Time Processing } \\
\text { RASF (Secs) }\end{array}$ \\
\hline Slices & 7757 & 224 & Conventional & 12.6 \\
\hline Look-up tables & 6583 & 665 & PC-based & \\
\hline Flip-Flops & 5296 & 196 & FPGA/ARM co-design & 3.37 \\
\hline Frequency $(\mathrm{MHz})$ & 518 & 352 & of the RASF algorithm & \\
\hline
\end{tabular}

In the second case, the same RASF algorithm was implemented using the proposed hybrid FPGA/ARM co-design architecture with the specialized SAs accelerator coprocessors. Particularly, the implementation of the RASF algorithm using the proosed architecture takes only 3.37 seconds for the large-scale $\mathrm{RS}$ image reconstruction in contrast to 12.6 seconds required with the $\mathrm{C}++$ implementation.

\section{Conclusions}

The principal result of this study relates to the efficient implementation of SAs hardware accelerators, which are integrated in a FPGA/ARM co-design scheme for the real-time enhancement/reconstruction of large-scale remote sensing (RS) imaging. With the proposed architecture, the corresponding RASF algorithm was executed in a real time computational mode (the 'real-time' being understood in a context of conventional RS users). We do believe that pursuing the aggregation of HPEC and SA techniques one could definitely approach the largescale real-time image processing requirements while performing the reconstruction of real-world hyperspectral RS imagery.

Acknowledgments. This study was supported by Consejo Nacional de Ciencia y Tecnología (México) under grant 51234-CB-2010-01.

\section{References}

[1] Shkvarko, Y.V.: Unifying Regularization and Bayesian Estimation Methods for Enhanced Imaging with Remotely Sensed Data. Part I Theory. IEEE Transactions on Geoscience and Remote Sensing 42, 923-931 (2004)

[2] Wehner, D.R.: High-Resolution Radar, 2nd edn. Artech House, Boston (1994)

[3] Castillo Atoche, A., Torres, D., Shkvarko, Y.V.: Towards Real Time Implementation of Reconstructive Signal Processing Algorithms Using Systolic Arrays Coprocessors. Journal of Systems Architecture 56(8), 327-339 (2010b)

[4] Kung, S.Y.: VLSI Array Processors. Prentice-Hall, NY (1998) 